\title{
SUI GRUPPI FINITI DI SOSTITUZIONI LINEARI.
}

\author{
Nota di G. Viranti (Pavia).
}

Adunanza del 12 gennajo 1913.

I. Il problema della determinazione di tutti $\mathrm{i}$ gruppi finiti di sostituzioni lineari d'una variabile è stato da tempo risolto in varî modi ${ }^{\mathrm{I}}$ ). Il seguente metodo, affatto elementare e indipendente da considerazioni cinematiche, puó forse presentare qualche interesse, almeno dal punto di vista didattico.

2. Affinchè una sostituzione lineare $\left(\begin{array}{l}\alpha \beta \\ \gamma \delta\end{array}\right)$, dove $\alpha \delta-\beta \gamma=\mathrm{I}$, sia ellittica $\left.{ }^{2}\right)$, è necessario e sufficiente che $\frac{\alpha+\delta}{2}$ sia reale $e$, in valore assoluto, minore di $\mathrm{I}$.

Che sia sufficiente, è cosa nota ${ }^{3}$ ).

Posto ora $\frac{\alpha+\delta}{2}=k$, e ricordando che $\left.{ }^{4}\right)$ :

$$
\theta=\left(k-\sqrt{k^{2}-1}\right)^{2}
$$

si ha, se la sostituzione è ellittica:

da cui :

$$
\left|k-\sqrt{k^{2}-1}\right|=\mathbf{I}
$$

e quindi:

$$
\left|k+\sqrt{k^{2}-\mathrm{I}}\right|=\frac{\mathbf{I}}{\left|k-\sqrt{k^{2}-\mathbf{I}}\right|}=\mathrm{I},
$$

$$
\left|k+\sqrt{k^{2}-\mathrm{I}}\right|=\left|k-\sqrt{k^{2}-\mathrm{I}}\right| \text {. }
$$

I due membri di questa eguaglianza sono le lunghezze delle diagonali d'un paral-

$\left.{ }^{1}\right)$ Vedi per es. : F. KLEIN, Ueber binäre Formen mit linearen Transformationen in sich selbst [Mathematische Annalen, t. IX (1876), pp. 183-208]; F. KIEIN, Vorlesungen über das Ikosaeder und die Aufösung der Gleichungen vom fünften Grade (Leipzig, Teubner, 1884); G. Favo, Ueber endliche Gruppen linearer Transformationen einer Veränderlichen [M nnatshefte für Mathematik und Physik, t. VII (I896), pp. 297-320] (dove si trova anche la letteratura dell'argomento).

2) Per la terminologia e le notazioni, vedi il mio manuale Hoepli : Elementi della teoria delle fun. zioni poliedriche e modulari (Milano, Hoepli, 1906).

3) Loc. cit. ${ }^{2}$ ), art. 24.

4) Loc. cit. $\left.{ }^{2}\right)$, art. 23. 
lelogramma avente come lati contigui $i$ vettori $k, \sqrt{k^{2}-1}$; affinchè esse sieno eguali, il parallelogramma dev'essere rettangolo, e quindi dev'essere reale il rapporto:

$$
\frac{i \sqrt{k^{2}-1}}{k}=\frac{\sqrt{\mathrm{I}-k^{2}}}{k}=\sqrt{\frac{\mathrm{I}}{k^{2}}-\mathrm{I}} \text {. }
$$

Ne segue che $k^{2}$ è reale, positivo e minore di I.

3. Affinchè il prodotto di due sostituzioni ellittiche sia una sostituzione ellittica, è necessario che $i$ poli delle due sostituzioni stiano sopra un cerchio.

Abbiansi le sostituzioni ellittiche $S_{s}, S_{2}$, poste sotto la forma:

$$
\frac{z^{\prime}-p_{1}}{z^{\prime}-q_{1}}=\theta_{1} \frac{z-p_{1}}{z-q_{1}}, \quad \frac{z^{\prime}-p_{2}}{z^{\prime}-q_{2}}=\theta_{2} \frac{z-p_{2}}{z-q_{2}},
$$

dove $\theta_{1}=e^{2 i \phi_{1}}, \theta_{2}=e^{2 i \phi_{2}}$, e $\phi_{1}, \phi_{2}$ sono reali. Se si rappresenta con $\left(\begin{array}{l}\alpha \beta \\ \gamma \delta\end{array}\right)$, dove $x \delta-\beta \gamma=\mathrm{I}$, la sostituzione $S_{1} S_{2}$, e se si pone:

si trova:

$$
\frac{\left(p_{1}-p_{2}\right)\left(q_{1}-q_{2}\right)}{\left(p_{1}-q_{2}\right)\left(q_{1}-p_{2}\right)}=\lambda, \quad \frac{1+\lambda}{1-\lambda}=\mu
$$

$$
\begin{aligned}
\frac{x+\delta}{2} & =\frac{\left(1+\theta_{1} \theta_{2}\right)-\lambda\left(\theta_{1}+\theta_{2}\right)}{2(1-\lambda) \sqrt{\theta_{1} \theta_{2}}}=\frac{\cos \left(\varphi_{1}+\varphi_{2}\right)-\lambda \cos \left(\varphi_{1}-\varphi_{2}\right)}{I-\lambda} \\
& =\cos \varphi_{1} \cos \varphi_{2}-\mu \operatorname{sen} \varphi_{1} \operatorname{sen} \varphi_{2} .
\end{aligned}
$$

Poichè $\frac{\alpha+\delta}{2}, \varphi_{1}, \varphi_{2}$ sono reali, lo è pure $\mu$, e quindi $\lambda$; e i 4 punti $p_{1}, q_{1}$, $p_{2}, q_{2}$, avendo rapporto anarmonico reale, stanno sopra un cerchio ${ }^{5}$ ).

4. Se due sostituzioni (ellittiche) appartengono ad un gruppo finito, $i$ loro poli, $i$ quali stanno sopra un cerchio, si separano reciprocamente. Ciò equivale a dire che $\lambda<0$, ossia che $|\mu|<\mathrm{I}$.

Se il prodotto delle due sostituzioni si rappresenta al solito sotto la doppia forma:

si ha $\left.{ }^{6}\right)$ :

$$
\begin{aligned}
& z^{\prime}=\frac{\alpha z+\beta}{\gamma z+\delta} \quad(\alpha \delta-\beta \gamma=1), \\
& \frac{z^{\prime}-p}{z^{\prime}-q}=\frac{z-p}{z-q} \quad\left(\theta=e^{2 i \varphi}\right),
\end{aligned}
$$

e quindi:

$$
\cos \varphi=\frac{\alpha+\delta}{2}
$$

$$
\mu=\frac{\cos \varphi_{1} \cos \varphi_{2}-\cos \varphi}{\operatorname{sen} \varphi_{1} \operatorname{sen} \varphi_{2}}
$$

5) Vedi: L. Bianchi, Lezioni sulla teoria delle funzioni di variabile complessa e delle funzioni ellittiche (Pisa, Spoerri, I90I), $\$ Io. Del resto basta osservare che l'argomento di $\lambda$ è, a meno di multipli di $2-$, la differenza degli angoli $q_{2} p_{1} p_{2}, q_{2} q_{1} p_{2}$, la quale è zero o $\pi$, se i 4 punti stanno sopra un cerchio [cfr. G. FANO, loc. cit. $\left.{ }^{\mathrm{I}}\right)$ ].

6) Loc. cit. ${ }^{2}$ ), art. $6 \mathrm{r}$. 
Ricordiamo che i soli possibili gruppi finiti di sostituzioni lineari sono quelli compresi nella tabella seguente ${ }^{7}$ ):

\begin{tabular}{|c|c|c|c|c|c|}
\hline & $r$ & $v_{1}$ & $v_{2}$ & $v_{3}$ & $n$ \\
\hline I & 2 & $n$ & $n$ & & $n$ \\
II & 3 & 2 & 2 & $m$ & $2 m$ \\
III & 3 & 2 & 3 & 3 & I 2 \\
IV & 3 & 2 & 3 & 4 & 24 \\
V & 3 & 2 & 3 & 5 & 60 \\
\hline
\end{tabular}

I poli di ciascun gruppo si distribuiscono in sistemi di $\frac{n}{v_{i}}(i=1, \ldots, r)$ poli equivalenti. Quindi i gruppi del tipo I hanno due soli poli, e ad essi non è applicabile il teorema da dimostrarsi.

Un gruppo del tipo II contiene un sottogruppo ciclico d'ordine $m$, e le rimanenti sostituzioni sono tutte d'ordine 2. Deve quindi il prodotto d'una sostituzione d'ordine 2 per una d'ordine $m$ essere d'ordine 2 , cioè dev'essere $\varphi=\frac{\pi}{2}$ per $\varphi_{1}=\frac{\pi}{2}, \varphi_{2}=\frac{\pi}{m}$. Ne segue $\mu=0, \lambda=-I$; i 4 poli formano un gruppo armonico.

Per i tipi III, IV, $\mathrm{V} \varphi_{1}, \varphi_{2}, \varphi$ possono prendere soltanto $\mathrm{i}$ valori:

$$
\frac{a \pi}{3}(a=\mathrm{I}, 2), \quad \frac{b \pi}{4}(b=\mathrm{I}, 2,3), \quad \frac{c \pi}{5}(c=\mathrm{I}, 2,3,4) .
$$

Osservando poi che ad un gruppo appartengono, insieme ad una sostituzione, tutte le sue potenze, si vede che, posto $\varphi_{i}=\frac{l_{i} \pi}{h_{i}}$, dove $h_{i}=3,4 \circ 5$, se $b_{i}=4$ e $l_{i} \neq 2$, la (I) deve sussistere, mutando eventualmente $\varphi$, anche per $l_{i}=2$; se $b_{i} \neq 4$, qualunque sia $l_{i}$, la (I) deve sussistere per $l_{i}=\mathrm{I}, \ldots, b_{i}-\mathrm{I}$. Quindi:

per $b_{1}=b_{2}=4$, si ha :

$$
\mu=-\cos \varphi
$$

per $b_{1}=4, b_{2}=3 \circ 5$ si ha, prendendo $l_{2}=\mathrm{I} \circ l_{2}=2$ :

$$
\mu=-\frac{\cos \varphi}{\operatorname{sen} \frac{\pi}{3}} \quad \text { o } \mu=-\frac{\cos \varphi}{\operatorname{sen} \frac{2 \pi}{5}}
$$

7) Loc. cit. ${ }^{2}$ ), art. 37 
per $b_{1} \neq 4, b_{2} \neq 4$ si ha, prendendo $l_{1}=l_{2}=\mathrm{I}$ o $l_{1}=\mathrm{I}, l_{2}=b_{2}-\mathrm{I}$ :

(4)

$$
\mu=\frac{\cos \frac{\pi}{h_{1}} \cos \frac{\pi}{h_{2}}-\cos \varphi}{\operatorname{sen} \frac{\pi}{h_{2}} \operatorname{sen} \frac{\pi}{h_{2}}}, \quad \mu=\frac{-\cos \frac{\pi}{b_{1}} \cos \frac{\pi}{h_{2}}-\cos \varphi}{\operatorname{sen} \frac{\pi}{h_{1}} \operatorname{sen} \frac{\pi}{h_{2}}},
$$

dove $\varphi$ puó essere diverso nelle due formole.

Ora il massimo valore di $|\cos \varphi|$ è $\cos \frac{\pi}{5}=\operatorname{sen} \frac{3 \pi}{10}=\frac{1+\sqrt{5}}{4}$, quindi dalle (2), (3) risulta immediatamente $|\mu|<$ I, e le (4) danno:

$$
|\mu| \leqslant \frac{\operatorname{sen} \frac{3 \pi}{10}-\cos \frac{\pi}{h_{1}} \cos \frac{\pi}{b_{2}}}{\operatorname{sen} \frac{\pi}{h_{1}} \operatorname{sen} \frac{\pi}{h_{2}}}=\frac{\frac{I+\sqrt{5}}{4}-C}{S}=H .
$$

Per le varie combinazioni di valori di $b_{1}, b_{2}$ si ha:

per $b_{1}=3, b_{2}=3$ :

$$
C=\frac{\mathrm{I}}{4}, \quad S=\frac{3}{4}, \quad H=\frac{\sqrt{5}}{3}<\mathrm{I} \text {; }
$$

per $b_{1}=3, b_{2}=5$ :

$$
C=\frac{I+\sqrt{5}}{8}, \quad S=\frac{I}{8} \sqrt{30-6 \sqrt{5}}, \quad H=\sqrt{\frac{3+\sqrt{5}}{15-3 \sqrt{5}}}<I
$$

per $b_{1}=5, b_{2}=5$ :

$$
C=\frac{3+\sqrt{5}}{8}, \quad S=\frac{5-\sqrt{5}}{8}, \quad H=\frac{\mathrm{I}}{\sqrt{5}}<\mathrm{I} .
$$

In tutti $\mathrm{i}$ casi dunque $|\mu|<\mathrm{I}$, quindi $\lambda<0$.

Le corde $p_{1} q_{1}, p_{2} q_{2}$ si tagliano pertanto in un punto interno $r$ del cerchio dei 4 poli.

5. I segmenti che congiungono le coppie di poli delle sostituzioni d'un gruppo finito banno tutti un punto comune.

Sieno $p_{3}, q_{3}$ i poli d'una terza sostituzione appartenente al gruppo finito considerato. I punti $p_{1}, q_{1}, p_{3}, q_{3}$ staranno sopra un cerchio, e $i$ punti $p_{2}, q_{2}, p_{3}, q_{3}$ staranno sopra un cerchio; il punto $r$ avrà egual potenza rispetto ai tre cerchi, e quindi $p_{3} q_{3}$ passerd per $r$.

6. Da ogni gruppo finito di sostituzioni lineari si ottiene per proiezione stereografica un gruppo finito di rotazioni d'una sfera su sè stessa; e quindi non vi sono altri gruppi finiti di sostituzioni lineari che $\mathrm{i}$ gruppi ciclici e poliedrici.

Basta condurre per $r$, perpendicolarmente al piano della variabile complessa, un 
segmento ro tale che:

$$
\overline{r o}^{2}=p_{\mathrm{r}} r \cdot r q_{\mathrm{s}}=p_{2} r . r q_{2}=\cdots,
$$

e prendere $r$ come centro ed or come raggio della sfera, ed $o$ come centro della projezione stereografica; gli angoli $p_{i} o q_{i}$ risultano retti, e quindi le proiezioni di $p_{i}$ e $q_{i}$ sulla sfera sono gli estremi d'un diametro ${ }^{8}$ ).

Pavia, 26 dicembre 1912.

G. VIVANTI. 